\title{
Médiévales
}

Langues, Textes, Histoire

$64 \mid$ printemps 2013

Temporalités de l'Égypte

Joan GREATREX, The English Benedictine Cathedral

Priories: Rule and Practice, ca 1270-ca 1420

Oxford, Oxford University Press, 2011, 544 p.

\section{Harmony Dewez}

\section{(2) OpenEdition}

Journals

Édition électronique

URL : https://journals.openedition.org/medievales/7036

DOI : $10.4000 /$ medievales. 7036

ISSN : 1777-5892

Éditeur

Presses universitaires de Vincennes

Édition imprimée

Date de publication : 1 juillet 2013

Pagination : 198-200

ISBN : 978-2-84292-371-6

ISSN : 0751-2708

Référence électronique

Harmony Dewez, "Joan greatrex, The English Benedictine Cathedral Priories : Rule and Practice, ca 1270ca 1420 », Médiévales [En ligne], 64 | printemps 2013, mis en ligne le 09 septembre 2013, consulté le 22 avril 2022. URL : http://journals.openedition.org/medievales/7036 ; DOI : https://doi.org/10.4000/ medievales.7036

Ce document a été généré automatiquement le 22 avril 2022.

Tous droits réservés 


\title{
Joan GREATREX, The English Benedictine Cathedral Priories: Rule and Practice,
} ca 1270-ca 1420

Oxford, Oxford University Press, 2011, 544 p.

\author{
Harmony Dewez
}

Dans l'Angleterre médiévale, les prieurés cathédraux sont des communautés bénédictines rattachées à une église cathédrale et dont l'abbé théorique est l'évêque. Neuf des seize cathédrales anglaises sont dans ce cas : Bath, Canterbury, Coventry, Ely, Norwich, Rochester, Winchester et Worcester dans la province ecclésiastique de Canterbury; Durham dans la province de York. Bath et Coventry ont en outre la particularité d'être des sièges doubles, le prieuré cathédral étant associé à un chapitre cathédral, respectivement à Wells et à Lichfield. Ces prieurés ont des origines diverses. Dans les cathédrales de Canterbury et Worcester, la présence monastique est attestée au $\mathrm{x}^{\mathrm{e}}$ siècle, tandis qu'à Winchester elle remonte même au vil ${ }^{\mathrm{e}}$ siècle. Au contraire, Bath, Coventry et Ely sont à l'origine des abbayes anglo-saxonnes, élevées au statut de cathédrale seulement au début du XII ${ }^{e}$ siècle. Enfin, les cathédrales monastiques de Durham et Norwich sont fondées telles quelles à la fin $\mathrm{du}_{\mathrm{XI}}^{\mathrm{e}}$ siècle, dans le mouvement qui suit la Conquête, tandis que la cathédrale de Rochester reçoit une communauté monastique à la même époque. D'un point de vue documentaire, la transformation par Henri VIII de la plupart de ces prieurés en chapitres cathédraux lors de la dissolution des monastères a permis une relativement bonne conservation de leurs archives, que Joan Greatrex avait déjà mise à profit en publiant en 1997 un travail prosopographique d'une ampleur considérable, reposant sur le dépouillement minutieux des comptes, chartes, cartulaires et registres divers de ces prieurés cathédraux depuis la Conquête, et dont le présent ouvrage est la suite ${ }^{1}$. Desservis par une réputation d'ordre en décadence, les Bénédictins d'après 1300 ont longtemps fait figure de parents pauvres de l'histoire ecclésiastique. Citant B. Thompson ou A.G. Dickens, l'auteur souligne le travail accompli ces dernières décennies pour redessiner l'histoire ecclésiastique des siècles ultimes du Moyen Âge et s'inscrit, avec d'autres tels que Barbara Harvey, dans une 
entreprise déjà ancienne d'étude de la vie monastique dans l'esprit de l'histoire des mentalités.

2 Pour J. Greatrex, l'étude des neuf prieurés cathédraux permet de travailler plus en profondeur que s'il s'était agi de traiter l'ensemble des monastères bénédictins, tout en conservant une forte dimension comparatiste. Dans le souci de faciliter une généralisation partielle aux autres monastères de cet ordre, elle commence par analyser les problèmes propres aux prieurés cathédraux et ce n'est qu'en conclusion qu'elle introduit des comparaisons avec d'autres établissements. Ces problèmes se cristallisent largement autour des rapports avec l'évêque et abbé titulaire, et notamment l'étendue des droits de ce dernier à intervenir dans les affaires des moines. Chaque siège opte pour des solutions indépendamment des autres; il y a bien sûr des tendances, mais celles-ci sont toujours très inégalement suivies. Par exemple, la division définitive du patrimoine entre l'évêque de Winchester et son prieuré de SaintSwithun n'est fixée qu'en 1284, alors qu'à Durham William de Saint-Calais l'aurait instaurée durablement dès son arrivée en 1083. Le droit de l'évêque à nommer le prieur - sujet majeur de conflit - fait l'objet d'un arrangement précoce de l'évêque avec les moines de Worcester en 1224, tandis que plusieurs prieurés gagnent progressivement le droit d'élire leur prieur au cours du xIII ${ }^{\mathrm{e}}$ siècle, époque à laquelle les prieurés cherchent à acquérir une autonomie toujours plus grande par rapport à leur évêque. L'église, à la fois monastique et cathédrale, reste, du fait de la séparation physique du palais épiscopal et des bâtiments du prieuré, le principal lieu de la contention, notamment lors des principales fêtes. D'autres points, comme l'admission des novices ou la nomination des obédienciers, sont également des sources de conflit et sont traitées au fil de l'ouvrage.

3 Les bornes de cette étude correspondent en amont à l'époque à partir de laquelle nous sont parvenus les comptes des obédienciers - les officiers monastiques - qui sont très riches en renseignements sur la vie quotidienne des moines. En aval, la date de 1421 correspond à la convocation par Henri $\mathrm{V}$ d'une importante assemblée des dignitaires bénédictins à Westminster afin de mettre en place une réforme. Si le cadre des prieurés cathédraux est strictement respecté, le cadre chronologique est souvent dépassé, aux deux bornes, tantôt grâce à l'ampleur et à la richesse de la réflexion de l'auteur, tantôt à cause des faiblesses de la documentation sur tel ou tel aspect. En effet, malgré la bonne conservation relative des archives de ces prieurés, il n'en reste pas moins de très importantes lacunes qui gouvernent les éclairages et les zones d'ombre. Pour saisir la « mentalité » des moines - elle cite Jacques Le Goff - $\mathrm{M}^{\text {me }}$ Greatrex choisit de détailler pas à pas les étapes de leur vie, de leur entrée dans le prieuré à leur décès en passant par les années de noviciat et d'apprentissage, entrant à chaque fois dans les détails les plus concrets de la vie quotidienne, de la répartition des tâches dans la communauté, des circulations de personnes dans et hors de l'enceinte, des repas, des habits, des rites, etc. Ce choix gouverne le plan de l'ouvrage, qui aborde ces différents thèmes suivant les âges auxquels ils correspondent. Les deux premiers chapitres traitent de la période qui va de l'admission à la profession et de la profession à l'ordination, avec entre autres un travail particulièrement intéressant sur l'âge des moines lors de ces étapes, la formation intellectuelle des novices et la routine monastique.

4 Idéalement, le candidat est d'abord examiné par le prieur et trois ou quatre moines afin d'évaluer ses compétences en chant, lecture, grammaire, dont il devait avoir une connaissance "suffisante». L'âge minimum pour l'admission est fixé par le chapitre 
bénédictin de 1278 à dix-huit ans, ce qui semble avoir été le plus souvent suivi. S'il est accepté par le prieur et l'évêque, il reçoit ensuite un "trousseau du moine " (W.A. Pantin) lors de la cérémonie d'admission, puis commence son année de probation au cours de laquelle il continue ses études. C'est lors de la profession qu'il reçoit le froccus et la cuculla qui composent l'habit monastique définitif. La suite est variable, car il est difficile d'estimer la proportion de moines ordonnés. À Durham et Canterbury, on arrive à inclure environ vingt et vingt-cinq pour cent des moines dans ce groupe, et l'ordination a lieu après respectivement moins de trois et entre trois et cinq années suivant la profession. Les sources d'information sont cependant très inégales. Le troisième chapitre, passant aux " années de maturité et de responsabilités ", traite en détail des officiers monastiques - les obédienciers - et de leurs fonctions au sein de la communauté. La vieillesse, l'infirmité et la mort sont traitées dans le cinquième et dernier chapitre, tandis que le quatrième se désolidarise de la logique par âges pour aborder le rythme de l'année liturgique.

5 L'étude synthétique d'un sujet aussi vaste est d'autant plus un tour de force que les prieurés cathédraux, comme les monastères, ont conservé leurs coutumes propres après Latran IV et la constitution des provinces bénédictines: chaque thème, de l'organisation des obédiences au déroulement des saignées, est ainsi l'occasion d'une comparaison des pratiques et d'une mise en valeur des particularités de chaque communauté. Il convient de souligner en particulier le travail minutieux que l'auteur produit sur le vocabulaire, notamment en ce qui concerne la hiérarchie des moines et novices, professés ou non. Son travail prosopographique, que nous avons déjà signalé, est largement mis à profit dans ses estimations des âges possibles des moines lors des différentes étapes de leur carrière, rendant particulièrement vivant le tableau qu'elle en dresse. Enfin, l'auteur a pris le parti de développer plus que d'autres la question de la formation intellectuelle des moines et de leurs lectures, comparant patiemment les listes d'ouvrages disponibles selon les époques et consacrant des annexes aux ouvrages de grammaire et d'histoire. Le résultat d'ensemble est une somme unique par l'ampleur du travail archivistique sur lequel il repose, abordant quantité d'aspects de la vie des moines. Le paysage brossé par cette multitude de touches est vivant, foisonnant de détails, et dévoile un monachisme qu'elle se garde de juger trop sévèrement.

\section{NOTES}

1. J. GREATREX, Biographical Register of the English Cathedral Priories of the Province of Canterbury, ca 1066-1540, Oxford, 1997. 